\title{
Aurora Retrive - An Augmented Reality Game using Real World Maps
}

\author{
Anupama.C.G, Gobinath.V, Satyajit Vijay Patil V
}

\begin{abstract}
The game developed uses real world map data to generate real world $3 D$ environment in Augmented Reality (AR). This Real world Game developed represents a way of technology implemented in augmented reality which is used to play Multi player Role Play Game (RPG). The game can be played on two different modes like role playing or multi-player mode. This game system is made as multiplayer game on a role playing environment for specific player, in which every player has their own role playing in it. The entire real world game environment is loaded in AR on any of the object that acts as the marker in the real world environment. This game system is developed using the UNITY game engine for creating game environment and the environment itself is created using real world data obtained using Map box. Vuforia studio frame work is used for implementation of game environment in AR. C\# programming language is used to program game play actions and environment generations and modifications in runtime.
\end{abstract}

Index Terms: Augmented Reality, Real World Map Data, Multiplayer RPG, Ray Casting.

\section{INTRODUCTION}

Game designers often use real world maps for Game Design. Simulation games like flight simulators employ real world maps but designers have to manually translate real world data to create games.

This game aims at exploring the use of map data to generate content at runtime for the games and how players can interact with the generated content. The challenges faced are: (1) modifying the data (how to transform the obtained data), (2) Adding the data (obtained data are always not complete like incomplete buildings data in most of the cities and only few select cities have complete data), (3) how to make the generated content and world interactive, (4) how to augment the generated content.

Augmented reality is visualization of the perspective of the real world environment in $3 \mathrm{D}$ computer generated environment where every object in the augmented reality has its own transforms of size and shape. Games are the one of the best way of implementing AR to its limit. This paper explains about the augmented reality game based on using marker. Marker based AR is used in order to keep the entire generated

Revised Manuscript Received on July 05, 2019.

Anupama.C.G, Assistant Professor,Department of Software Engineering, SRMIST, Chennai, India

Gobinath.V, SRMIST, Chennai , India

Satyajit Vijay Patil V, SRMIST, Chennai, India environment in single place so the player can move only around the marker, in order to provide safe play without making player to move over long distance.

Most of the games developed these day are either multiplayer( as multiplayer games motivate players to compete in a challenge together in a team to complete a task or against each other which increases social closeness of the players [9] ) or story based where player will have multi player or play in the story based game but not together. In this we have created a multiplayer game with story mode for each and every single player in the game. Every player will have same story to finish while they have to play as the multiplayer also.

Multi-player games are used to interact with other players in single game but a multiplayer game without story based is not going to involve in any complex task handling. The players of this game will play the multiplayer game for making up points and they have to play the story based game to make the missions and finish the game. Without finishing any one of the mode the game can't be completed because the RPG in integrated with multiplier mode.

\section{RELATED WORKS}

Out project builds upon the notions of Augmented Reality, Real world Map in games, Multiplayer. This section provides a brief overview of the above mentioned fields.

\section{A. Augmented Reality:}

A technology that superimposes a computer-generated image/3D Model on a user's view of the real world, thus providing a composite view [8, 7]. Vuforia [2] with Unity engine makes it easier to implement augmented reality concept in games and apps. One can choose between marker-based and marker less, marker-based uses a pre-determined target for tracking and to augment the virtual $3 \mathrm{~d}$ object in real world when it detects the pre-determined target, the target can be a single-image target, multi-image target or 3d model target. Marker less doesn't uses any pre-determined targets to track instead it uses real world itself to track and tracks the floor or plane surface to augment. But the marker less are supported only on few latest select devices, hence our project uses a marker-based target.

The gaming industry has already embraced AR technology and due to recent advances in AR SDK's/API's such as ARCORE [3] from google and AR Kit [4] from Apple number of AR Games have been emerging on the Internet for example The Machines [1]

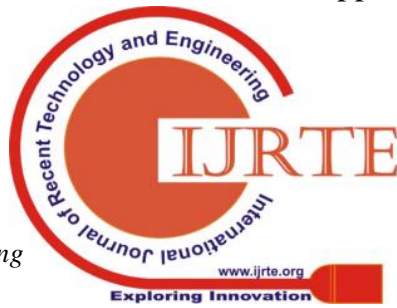


from AR Kit, and others such as AR air hockey, Titans of Space, collaborative combat against virtual enemies, and AR-enhanced pool table games.

\section{B.Real World Map in games:}

Games which game play/ game-content or based on external data or called as data games, these games support exploration from these data [6] .The API's such as Google Maps, Map box, Open Street Map provide relevant data for building a real world map. Games such as Pokémon GO, Ingress overlays game objects, tasks on the real world map where player has to move in real world to play the game, as the player moves the GPS updates the map in game at runtime. These maps aren't suitable for $3 \mathrm{~d}$ fps games as these are just a flat maps mostly 3D maps in games have been around for quite a few years, but the implementation of real world data are done manually, each type of data was translated manually into the game, thus only few select city/location maps can be built. For example True Crime: Streets of LA is based on LA map which is constructed manually by game designers. The API's such as map box and google maps can helps developers to implement real $3 \mathrm{~d}$ map of any location at runtime which developers can modify to make the map look realistic with their own scripts.

\section{C.Multiplayer:}

Multiplayer is a mode of play for computer games and video games where two or more gamers can play in the same game at the same time, co-operatively as a clan (or team) or head-to-head competitively (often referred to as death match) . Multiplayer mode may be a split screen where the gamers play at the same time on one system, or where gamers play on separate systems connected to a LAN or Internet game server. The game play is created by the host of the game. The host device acts as the server of the game.

\section{Methodology}

\section{A. Real World Map:}

Map box is used to obtain the data necessary to create a real world map at runtime for any location. The latitude, longitude data obtained user's GPS is sent to map box which then sends map data. The data that are sent by map box are $2 \mathrm{~d}$ map, roads, rails, buildings, woods (forest) and farmlands layers etc. These data are then converted into game objects [5] by map box-unity SDK. These translated game objects are then used to create a $3 \mathrm{~d}$ map with features like tress in park and forest, trains on rail tracks, buildings with textures , modified water bodies(lakes, water), farms in farmlands etc.

\section{B. Building Modification:}

The Buildings data obtained generates a mesh at location of building on the map with around 0.2 approximate height except for few select cities which are accurate and realistic. Hence when the data is obtained for an infamous city the data needs to be modified, the height of the buildings are modified to random height and the textures tilling is set according to the height of the building (Figure 1). without any 3D map data. The implementation of Real world

Figure 1. Modified height and textures of buildings.

\section{Parks, Forest and Agriculture Generation:}

On the park and wood layer trees are generated randomly. As the layers are converted into mesh, the size in $\mathrm{x}$ and $\mathrm{z}$ direction are calculated and center of the mesh is obtained, using these values a square shaped region is identified around the center of mesh for random generation of trees but the shape of mesh is often irregular as parks and forest are not square shaped in real life. To spawn trees within the irregular shape of mesh Ray casting is used (Ray casting definition), so to achieve this a for loop is iterated and in each iteration a random $\mathrm{x}$ and $\mathrm{z}$ coordinates are chosen with $\mathrm{y}$ being default to 10 and then a ray casting is initiated at $\mathrm{x}, \mathrm{y}$ and $\mathrm{z}$ coordinates to check if the ray hits the mesh a tree is generated else not (Figure 2). The result will be trees only generated on layer than in the square shaped region (Figure 3).

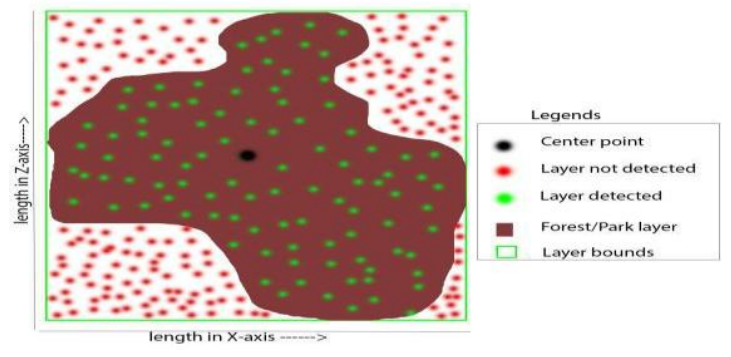

Figure 2. Illustrating the Ray casting process done to detect the forest/park layers

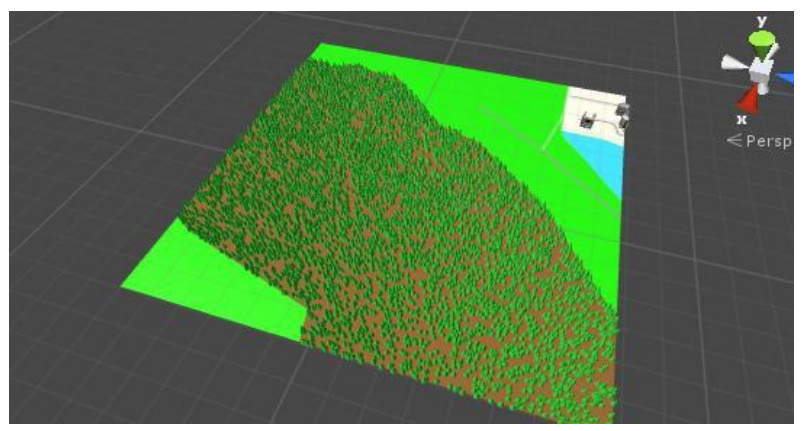

Figure 3. Image of generated trees on forest/park layer

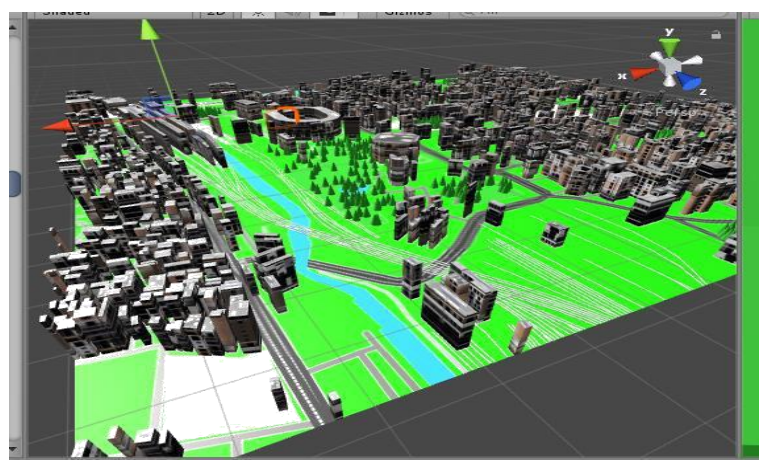

\section{Roads and Railways:}

The railway layers generates a line mesh for each tracks on the map (shown as white line in Figure 1). These line

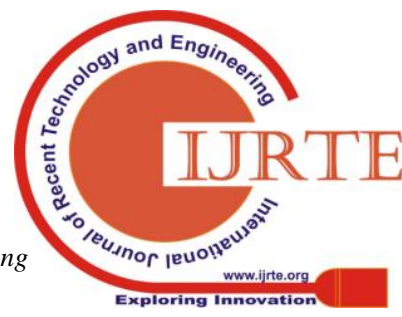


meshes are used to create path for a $3 \mathrm{~d}$ model Trains (transportation) to follow the rail track. Ray casting is used to generate points at each few unit distance where the Rail layer is detected. Rays are generated in an iterative manner until a track is detected, once detected $\mathrm{x}$ and $\mathrm{z}$ coordinates are incremented and decremented accordingly to detect the direction of the track and points are generated in the direction. These Points are then used to construct a Bezier curve, and the Bezier curve then generates a Rail tracks mesh and creates a path for the $3 \mathrm{D}$ train model along the curve to follow.

\section{E. Augmentation:}

In augmentation we use vuforia's marker-based single image target with extended tracking to augment the game in the real world and to augment each generated object at runtime Vuforia library functions are used to augment them in the Real world.

\section{F. Positioning Spawn points for player spawning in Generated Real World Map:}

Spawning players in runtime generated map in a fixed/default position might not also be possible always as it might be occupied by other runtime generated Game object sometimes, so a random point is chosen and cube is generated then it is checked if it intersects any other Game object or not, if intersects a new random point is selected and the cycle continues until it finds an empty/free space.

\section{G. Multiplayer:}

Unity multiplayer is implemented for multiplayer in the game. The game project allows up to 4 max connections were the players have to compete with each other to win. In multi-player they have to finish the game by playing the story programmed in multiplayer. Each and every player will have their own game play and they have to finish the story mode integrated with multiplayer to finish the game.

In multi-player game, the player's story and his game play ends if his game character is terminated in game. The game character disappears and the player will be no longer able to control the game character.

\section{H. Game UI:}

Game has the interface to control the game character. The left side joystick control is used to control the game character movement and Aim and the right side joystick control is used to fire the missile.

The game character has the health bar always floating on top of it. This health bar decreased in negative value of 1 when the enemy fire at the character. When the health bar is reduced to zero, the game character has no health and it destroys. The health bar is fixed at 100 as the fixed value for every player inn game.

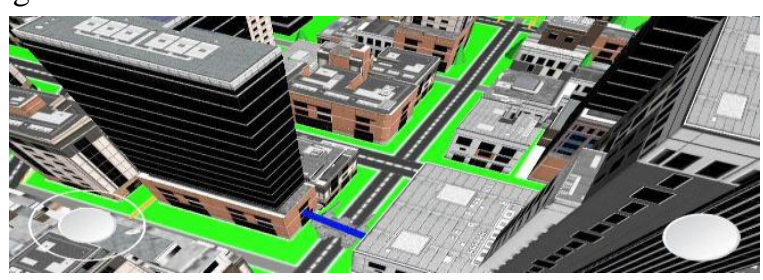

Figure 4: Game play.

The UI for Multiplayer connection is shown in the (figure 5). The UI asks the player whether to join the game that someone else has hosted or to host the multiplayer game. If the game is hosted by the player other players joining in this game should connect to the host player's internet connection and connect to the IP of the host. Every player has to connect to the IP for mobile device is 192.168.43.1 of game host. After entering the host the join button should be clicked and tat loads the match making UI where every player will be listed.
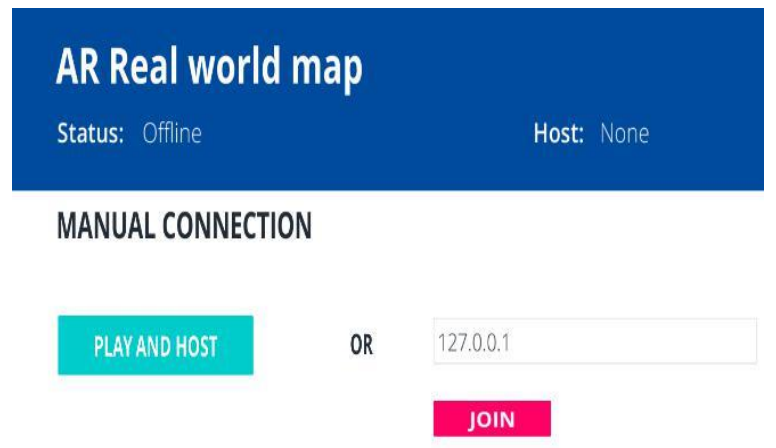

Figure 5: Multiplayer connection

\section{Game AI:}

Game AI is used for the Story mode that is intenerated with multiplayer. Game AI generate random enemies and random points at the map which common enemy for all the players. The enemies generated by the Game AI has a health level. After the health level reaches zero the enemy is destroyed.

The enemy AI after generated at random points, it will be moving randomly unless its target is found within its destination. If the AI finds any target in destination it follows the target and fires the missiles to attack the target. Every player in the multiplayer game act as the target for Game AI.

\section{J. Game working process:}

The player has to choose the process whether he is going to host the game for multiple players or he is going to join the hosted game. This selection process is maintained by the unity network manager. After choosing either one of the process unity network manager establishes the connection to the game manager. Game manager initializes the game objects such as player buildings and map for the first time and generates it.

Then the game manager initializes vuforia for augmenting the generated objects. Vuforia initializes plane finder. Plane finder accesses the camera and tracks the available plane which is image target .If any plane is detected vuforia augments the game objects over that plane. In this game a specific image is used an image target for augmenting the game objects.

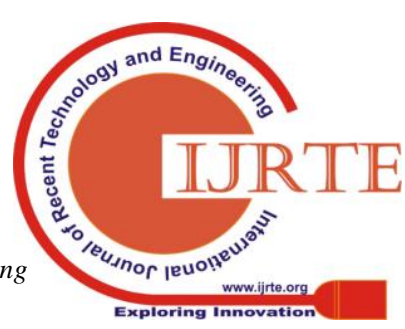




\section{Conclusion}

A game which can create a playable map for any Real world location and augment the game environment the game environment in real world with multiple players. This game incudes of Role playing integrated with Multiplayer environment. The map and buildings loaded in real-time based on the real world data obtained from Map box.

\section{REFERENCES}

1. TheMachines:http://www.themachinesgame.com

2. Vuforia: https://www.vuforia.com/

3. ARCORE: https://developers.google.com/ar/

4. ARKIT: https://developer.apple.com/arkit/

5. GameObjects:https://docs.unity3d.com/560/Documentation/Manual/Ga meObjects.html

6. M. Gustafsson Friberger, J. Togelius, A. Borg Cardona, M. Ermacora, A. Mousten, M. M. Jensen, V.-A. Tanase, and U. Brøndsted. Data games. "In Proceedings of the Workshop on Procedural Content Generation", 2013.

7. Chek Tien Tan, Donny Soh, "Augmented Reality Games: A Review" in Proceedings of the Asian Simulation and AI in Games Conference, GAMEON-ASIA, EUROSIS, Volume: 2, April 2011.

8. Vaishali Agrawal, Jignesh Patel, "A REVIEW: AUGMENTED REALITY AND ITS WORKING”, International Research Journal of Engineering and Technology (IRJET) e-ISSN: 2395 -0056, Volume: 04 Issue: 05 | May -2017.

9. Iro Voulgari ; Vassilis Komis ; Demetrios G. Sampson, "Player Motivations in Massively Multiplayer Online Games”, 2014 IEEE 14th International Conference on Advanced Learning Technologies,7-10 July 2014.

10.LEIGH ACHTERBOSCH, ROBYN PIERCE, AND GREGORY SIMMONS School of Information Technology and Mathematical Sciences," Massively Multiplayer Online Role-Playing Games: The Past, Present, and Future",University of Ballarat, Australia ACM Computers in Entertainment, Vol. 5, No. 4, Article 9. Publication date: March 2008 